\title{
Thermocapillary flow in an annular liquid layer painted on a moving fiber
}

\author{
W.R. $\mathrm{Hu}^{\mathrm{a}, \mathrm{b}, *}$, N. Imaishi ${ }^{\mathrm{b}}$ \\ ${ }^{\mathrm{a}}$ Institute of Mechanics, Chinese Academy of Science, Beijing 100080, People's Republic of China \\ ${ }^{\mathrm{b}}$ Institute of Advanced Material Study, Kyushu University, Kasuga, Fukuoka 816-8580, Japan
}

Received 20 November 1999

\begin{abstract}
In the present paper, a theoretical model is studied on the flow in the liquid annular film, which is ejected from a vessel with relatively higher temperature and painted on the moving solid fiber. A temperature gradient, driving a thermocapillary flow, is formed on the free surface because of the heat transfer from the liquid with relatively higher temperature to the environmental gas with relatively lower temperature. The thermocapillary flow may change the radii profile of the liquid film. This process analyzed is based on the approximations of lubrication theory and perturbation theory, and the equation of the liquid layer radii and the process of thermal hydrodynamics in the liquid layer are solved for a temperature distribution on the solid fiber. (C) 2000 Elsevier Science Ltd. All rights reserved.
\end{abstract}

Keywords: Thermocapillary flow; Liquid film; Fiber processing

\section{Introduction}

Fluid coating on a fiber is an interesting subject for the fluid mechanics, and also for fiber processing. The usual model considers a moving solid fiber drawn from a liquid with half-infinite free surface, and the capillary force controls the thickness of the coating liquid [1]. In the present paper, the fluid or melt layer ejected from a fluid or melt vessel and coated on a fiber are considered to simulate the Barus effect or Die Swell effect, which changes the cross-section in a polymer jet and is often observed in the polymer processing. The effect of Newtonian fluid can only explain a small variation of cross-section, for example, an increment of

\footnotetext{
* Corresponding author.
}

$20 \%$, and the larger increment is explained usually by the rheological property of the liquid medium [2]. In fact, the property of polymer fluids varies from Newtonian to rheological in a large range. The polymer processing requires an understanding of the heat and mass transfer processes, and in addition, the effects of surface tension and its variation with temperature. The influence of surface tension on extradite swell shows that the surface tension decreases the extradite cross-section in a Newtonian fluid [3]. However, the solutal capillary flow induced by the surfactant of the liquid may increase the thickness of the liquid layer [4]. The temperature of liquid or melt ejected from the exit of a manufactured vessel is higher than the environmental gas temperature. The stronger heat exchange results a relatively larger temperature gradient on the free surface, especially in the region near the 


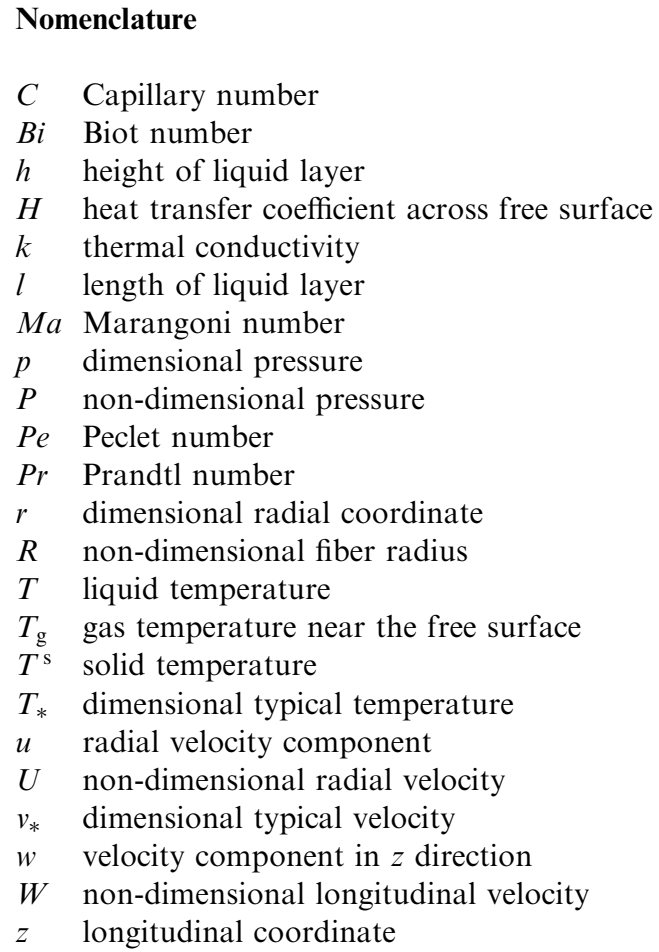

\section{Greek symbols}

$\varepsilon \quad$ geometrical aspect ratio

$\gamma \quad$ expanding angle of liquid layer

$\eta \quad$ non-dimensional height of liquid layer

$\kappa \quad$ thermal diffusivity

$v \quad$ kinematic viscosity

$\rho$ density of liquid

$\sigma \quad$ surface tension

$\xi \quad$ non-dimensional coordinate of $r$

$\zeta$ non-dimensional coordinate of $z$

$\Theta$ non-dimensional liquid temperature

$\Theta_{\mathrm{g}}$ non-dimensional gas temperature

$\Theta^{\mathrm{s}}$ non-dimensional solid temperature

$\Psi$ non-dimensional stream function

\author{
Subscripts \\ $e \quad$ at cross-section $z=0$ \\ $l \quad$ at cross-section $z=l$ \\ $i \quad$ at solid boundary $r=r_{i}$ \\ * dimensional typical value \\ $m$ at cross-section $z=l$ and solid boundary $r=r_{i}$
}

exit of vessel. Therefore, a thermocapillary flow will be induced in the liquid layer, and will change the cross-section of the liquid layer.

As the heat transfer process will result the nonuniformity of the temperature on the free surface of the liquid layer, the thermocapillary flow may be induced in a thin liquid layer on a planar solid wall with non-uniform temperature distribution. By using an approximation of the lubrication theory, a higher order ordinary differential equation of liquid thickness was demonstrated, and its solution for given temperature distribution at solid boundary was obtained for unsteady cases in a thin liquid layer [5]. A similar method was applied to discuss the steady cases where a smooth condition at the symmetric cross-section was imposed [6]. The Die Swell effect due to the thermocapillary flow in a jet of liquid film painted on a moving planar solid boundary was analyzed, and the results show that the thermocapillary flow may increase the cross-section of the liquid layer [7]. All these models deal with a motionless film with infinite extention [5,6] or a moving solid plane [7], and have a non-uniform temperature distribution on the solid plane. The variations of free surface curvatures are given in the longitudinal direction.
In the present paper, the change in cross-section due to the thermocapillary convection in an axisymmetric and steady model of Newtonian fluid is discussed for the case of jet annular liquid layer casting on a moving cylindrical fiber. The reason for using the Newtonian fluid assumption is that the effect of thermocapillary flow is emphasized specially, and furthermore, the coupling effect of both thermocapillary flow and rheological effect may be discussed in the next step. The lubrication approximation and the perturbation method are used. The variation in free surface curvature radius of fluid coating on a cylindrical fiber is mainly the radius of the liquid layer but not the curvature radius in the longitudinal direction as in the cases of $[5,6]$. The finite, but not very small, capillary number will be discussed in the present paper. An ordinary differential equation of second order for the radii of the liquid layer is demonstrated, and the solution is given by two conditions of the liquid radii. The solution shows clearly the important effect of thermocapillary convection on the thickness variation of the liquid layer, and the variation of the cross-section depends sensitively on the thermocapillary effect in addition to other typical parameters. 


\section{The model of jet annular liquid layer}

A simplified model of fluid coating on a fiber is suggested as shown in Fig. 1, where the liquid layer of height $h_{0}$ is ejected from the exit of a melt or liquid vessel and then attached on a moving solid fiber. The cylindrical coordinate system $(r, \theta, z)$ is adopted, and the axisymmetric process depending on $(r, z)$ is assumed, therefore, $\partial / \partial \theta=0$. The melt or liquid will flow to a distance $z=l$ far from the exit of the vessel at $z=0$, and the distance $l$ is much larger than the radius $r_{0}$. The geometrical aspect ratio $\varepsilon=r_{0} / l$ is small, i.e. $\varepsilon \ll 1$. The liquid temperature $T_{\mathrm{e}}$ at the exit is higher than the environmental gas temperature $T_{\mathrm{g}}$ and the temperature $T_{\mathrm{m}}$ at $z=l$. A moving solid fiber is drawn from the center of the liquid vessel, and moves with the same velocity $u_{0}$ of the liquid at the lower boundary of the exit. The heat transfer between moving solid fiber, liquid jet and environmental gas may result a large temperature gradient on the free surface, especially near the exit of the vessel, and then, the thermocapillary flow will be driven in addition to the jet flow.

The liquid is assumed to be incompressible and Newtonian with constant kinematic viscosity, $v$ and thermal diffusivity, $\kappa$. The relationships of the mass conservation, the momentum conservation and the energy conservation will be given as

$$
\begin{aligned}
& \frac{1}{r} \frac{\partial r u}{\partial r}+\frac{\partial w}{\partial z}=0 \\
& u \frac{\partial u}{\partial r}+w \frac{\partial u}{\partial z}=-\frac{1}{\rho} \frac{\partial p}{\partial r}+v\left(\frac{\partial^{2} u}{\partial r^{2}}+\frac{1}{r} \frac{\partial u}{\partial r}-\frac{u}{r^{2}}+\frac{\partial^{2} u}{\partial z^{2}}\right),
\end{aligned}
$$

$$
u \frac{\partial w}{\partial r}+w \frac{\partial w}{\partial z}=-\frac{1}{\rho} \frac{\partial p}{\partial z}+v\left(\frac{\partial^{2} w}{\partial r^{2}}+\frac{1}{r} \frac{\partial w}{\partial r}+\frac{\partial^{2} w}{\partial z^{2}}\right)
$$

$$
u \frac{\partial T}{\partial r}+w \frac{\partial T}{\partial z}=\kappa\left(\frac{\partial^{2} T}{\partial r^{2}}+\frac{1}{r} \frac{\partial T}{\partial r}+\frac{\partial^{2} T}{\partial z^{2}}\right),
$$

where $(u, 0, w)$ is the velocity vector and the azimuthal velocity is assumed to be zero, and $\rho, p$ and $T$ are, respectively, the density, pressure and temperature of the liquid, $v$ and $\kappa$ are, respectively, the kinematic viscosity and thermal diffusivity.

The boundary condition of the liquid layer can be summarized as follows.

$$
\begin{aligned}
& r=r_{i}: \quad u=0, \quad w=w_{0}, \quad T=T^{\mathrm{s}}(z) ; \\
& r=h(z): \\
& u=h^{\prime} w,
\end{aligned}
$$

$$
\begin{aligned}
& \frac{\rho v}{\left(1+h^{\prime 2}\right)^{1 / 2}}\left[\left(1-h^{\prime 2}\right)\left(\frac{\partial u}{\partial z}-\frac{\partial w}{\partial r}\right)+2 h^{\prime 2}\left(\frac{\partial u}{\partial r}-\frac{\partial w}{\partial z}\right)\right] \\
& =\sigma_{T}\left(\frac{\partial T}{\partial z}+h^{\prime} \frac{\partial T}{\partial r}\right),
\end{aligned}
$$

$$
\begin{aligned}
p= & \frac{2 \rho v}{1+h^{\prime 2}}\left[\left(\frac{\partial u}{\partial r}-h^{\prime} \frac{\partial w}{\partial r}\right)-h^{\prime}\left(-\frac{\partial u}{\partial z}+h^{\prime} \frac{\partial w}{\partial z}\right)\right] \\
& -\left[\sigma_{0}+\sigma_{r}^{\prime}\left(T-T_{*}\right)\right]\left[\frac{h^{\prime \prime}}{\left(1+h^{\prime 2}\right)^{3 / 2}}-\frac{1}{h\left(1+h^{\prime 2}\right)}\right],
\end{aligned}
$$

$-k \frac{\partial T}{\partial n}=H\left(T-T_{\mathrm{g}}\right)$

$z=0: \quad u=u_{e}(r), \quad w=w_{e}(r), \quad T=T_{e}(r)$

$z=l: \quad u=u_{l}(r), \quad w=w_{l}(r), \quad T=T_{l}(r)$,

where $r=h(z)$ is the equation of free surface and $r_{0}=h(0)$, the superscript "'" denotes the differential

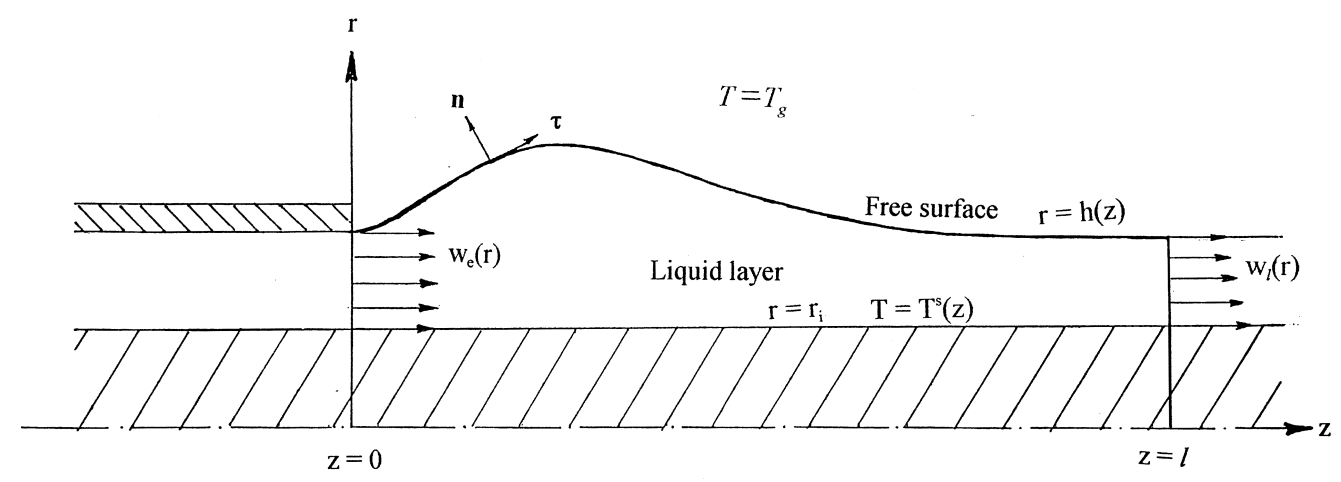

Fig. 1. The schematic diagram of the physical model of a jet liquid film. 
$h^{\prime}(z)=\mathrm{d} h(z) / \mathrm{d} z, \mathbf{n}$ is the unit vector in the normal direction, $r_{i}$ is the radius of cylindrical solid, $T_{*}$ is a constant reference temperature, denoting $w_{\mathrm{e}}\left(r_{i}\right)=$ $w_{l}\left(r_{i}\right)=w_{0}$ and $T_{l}\left(r_{i}\right)=T_{m}$, and the coefficients $k$ and $H$ are, respectively, the thermal conductivity of liquid and heat transfer coefficient across the free surface. Eq. (2.6) describes the free surface as a stream surface. Eq. (2.7) shows the viscose stress in the tangent direction balancing with the thermocapillary force driven by the surface tension gradient, and Eq. (2.8) is the momentum conservation in the normal direction. Eq. (2.9) gives the heat transfer across the free surface where the radiation effect is omitted.

The temperature $T^{\mathrm{s}}$ in the solid fiber will be solved together with the fields in the fluid and satisfies the Laplace equation

$\frac{\partial^{2} T^{\mathrm{s}}}{\partial r^{2}}+\frac{1}{r} \frac{\partial T^{\mathrm{s}}}{\partial r}+\frac{\partial^{2} T^{\mathrm{s}}}{\partial z^{2}}=0$

and the boundary conditions

$$
\begin{gathered}
\frac{\partial T^{\mathrm{s}}(0, z)}{\partial r}=0, \quad T^{\mathrm{s}}\left(r_{i}, z\right)=T\left(r_{i}, z\right), \\
k^{\mathrm{s}} \frac{\partial T^{\mathrm{s}}\left(r_{i}, z\right)}{\partial r}=k \frac{\partial T\left(r_{i}, z\right)}{\partial r},
\end{gathered}
$$

where $k^{\mathrm{s}}$ is the thermal conductivity of the solid fiber.

In principle, the thermocapillary flow in the liquid layer will be obtained by connecting the solution of fluid region, Eqs. (2.1)-(2.11), and the solution of solid region, Eqs. (2.12) and (2.13). A linear solution of $T^{\mathrm{s}}$ could be obtained as follows

$$
\frac{T^{\mathrm{s}}}{T_{*}}=1-\left(1-\frac{T_{m}}{T_{*}}\right) \frac{z}{\ell}
$$

where $T_{*}$ is a constant temperature, which is the highest temperature in the liquid region and relates to the temperature at $z=0$. By using the temperature solution, Eq. (2.14) in the solid fiber, the problem is then simplified, and may be solved in the fluid region only.

Non-dimensional quantities and parameters may be introduced based on the lubrication theory as follow [5].

$$
\begin{gathered}
\xi=\frac{r}{r_{0}} \quad \zeta=\frac{z}{\ell}, \quad \eta=\frac{h}{r_{0}}, \quad R=\frac{r_{i}}{r_{0}}, \quad \varepsilon=\frac{r_{0}}{\ell}, \\
U=\frac{u}{v_{*}}, \quad W=\frac{w}{v_{*}}, \quad \varepsilon^{2} P=\frac{\rho \ell}{\rho v v_{*}}, \quad \Theta=\frac{T}{T_{*}} \\
\Theta^{\mathrm{s}}=\frac{T^{\mathrm{s}}}{T_{*}},
\end{gathered}
$$

where $r_{0}$ is the radius of vessel at the exit $z=0$, the typical temperature $T_{*}$ and the typical velocity $v_{*}$ are adopted, respectively, as

$T_{*}=T^{\mathrm{s}}\left(r_{i}, 0\right), \quad v_{*}=-\varepsilon|\mathrm{d} \sigma / \mathrm{dT}| \mathrm{T}_{*} / \rho v$,

and $\sigma$ is the surface tension. The basic feature of the lubrication problem is that, there are two typical scales of different order of magnitude, that is, one typical scale $l$ is much larger than the $r_{0}$, and other quantities have different orders of magnitude. The non-dimensional parameters are, respectively, the Reynolds number, $R e$, and the Peclet number, $P e$, i.e.

$R e=\frac{v_{*} \ell}{v}, \quad P e=\frac{v_{*} \ell}{\kappa}$.

The Prandtl number, $P r$, and the Marangoni number, $M a$, are given as

$\operatorname{Re}=\operatorname{Pr} M a$,

where $\quad M a=-\sigma_{\mathrm{T}}^{\prime} T_{*} l / \kappa v=\varepsilon P e . \quad$ Non-dimensional equations are then written as follows.

$\frac{1}{\xi} \frac{\partial \xi U}{\partial \xi}+\frac{\partial W}{\partial \zeta}=0$

$$
\begin{gathered}
\operatorname{Re\varepsilon }^{4}\left(U \frac{\partial U}{\partial \xi}+W \frac{\partial U}{\partial \zeta}\right) \\
=-\frac{\partial P}{\partial \xi}+\varepsilon^{2}\left(\frac{\partial^{2} U}{\partial \xi^{2}}+\frac{1}{\xi} \frac{\partial U}{\partial \xi}-\frac{U}{\xi^{2}}\right)+\varepsilon^{4} \frac{\partial^{2} U}{\partial \xi^{2}}, \\
\operatorname{Re\varepsilon }^{2}\left(U \frac{\partial W}{\partial \xi}+W \frac{\partial W}{\partial \zeta}\right) \\
=-\frac{\partial P}{\partial \zeta}+\frac{\partial^{2} W}{\partial \xi^{2}}+\frac{1}{\xi} \frac{\partial W}{\partial \xi}+\varepsilon^{2} \frac{\partial^{2} W}{\partial \xi^{2}}, \\
P e \varepsilon^{2}\left(U \frac{\partial \Theta}{\partial \xi}+W \frac{\partial \Theta}{\partial \zeta}\right)=\frac{\partial^{2} \Theta}{\partial \xi^{2}}+\frac{1}{\xi} \frac{\partial \Theta}{\partial \xi}+\varepsilon^{2} \frac{\partial \Theta}{\partial \zeta^{2}}
\end{gathered}
$$

The corresponding boundary conditions will be:

$$
\begin{aligned}
& \xi=R: \quad W=W_{0}(\text { const }), \quad U=0, \quad \Theta=\Theta^{\mathrm{s}}(\zeta) \\
& \xi=\eta(\zeta): \\
& \eta(0)=1
\end{aligned}
$$

$U(\xi, \eta)=\eta^{\prime} W(\xi, \eta)$, 


$$
\begin{array}{lr}
\frac{\partial W}{\partial \xi}+\varepsilon^{2}\left[\left(\frac{\partial U}{\partial \zeta}-\eta^{\prime 2} \frac{\partial W}{\partial \xi}\right)+2 \eta^{\prime}\left(\frac{\partial U}{\partial \xi}-\frac{\partial W}{\partial \zeta}\right)\right. & U=\sum_{n=0}^{\infty} \varepsilon^{n} U^{(n)}, \quad W=\sum_{n=0}^{\infty} \varepsilon^{n} W^{(n)} \\
\left.-\varepsilon^{2} \eta^{\prime 2} \frac{\partial U}{\partial \zeta}\right] & \Theta=\sum_{n=0}^{\infty} \varepsilon^{n} \Theta^{(n)}, \quad \eta=\sum_{n=0}^{\infty} \varepsilon^{n} \eta^{(n)} \\
=-\sqrt{1+\varepsilon^{2} \eta^{\prime 2}}\left(\frac{\partial \Theta}{\partial \zeta}+\eta^{\prime} \frac{\partial \Theta}{\partial \xi}\right), & \text { Substituting Eq. (3.2) into the }
\end{array}
$$$$
P=\frac{1+C-C \Theta}{C}\left[-\frac{\varepsilon^{2} \eta^{\prime \prime}}{\left(1+\varepsilon^{2} \eta^{\prime 2}\right)^{3 / 2}}+\frac{1}{\eta\left(1+\varepsilon^{2} \eta^{\prime 2}\right)^{1 / 2}}\right]
$$$$
+\frac{2 \varepsilon^{2}}{1+\varepsilon^{2} \eta^{\prime 2}}\left[\left(\frac{\partial U}{\partial \xi}-\eta^{\prime} \frac{\partial W}{\partial \xi}\right)\right.
$$$$
\left.+\varepsilon^{2} \eta^{\prime}\left(-\frac{\partial U}{\partial \zeta}+\eta^{\prime} \frac{\partial W}{\partial \zeta}\right)\right]
$$

Substituting Eq. (3.2) into the governing equations and boundary conditions, the problem could be solved order by order.

The zero-order relationships may be written as

$\frac{1}{\xi} \frac{\partial \xi U^{(0)}}{\partial \xi}+\frac{\partial W^{(0)}}{\partial \zeta}=0$,

$\frac{\partial P^{(0)}}{\partial \xi}=0$

$\frac{\partial \Theta}{\partial \xi}-\varepsilon^{2} \eta^{\prime} \frac{\partial \Theta}{\partial \zeta}=-B i\left(\Theta-\Theta_{\mathrm{g}}\right)\left(1+\varepsilon^{2} \eta^{\prime 2}\right)^{1 / 2}$

$\zeta=0: \quad U=U_{\mathrm{e}}(\xi), \quad W=W_{\mathrm{e}}(\xi), \quad \Theta=\Theta_{\mathrm{e}}(\xi)$

$\zeta=l: \quad U=U_{l}(\xi), \quad W=W_{l}(\xi), \quad \Theta=\Theta_{l}(\xi)$

where the Capillary number, $C=-\sigma_{T}^{\prime} T_{*} / \sigma_{0}$, is not small, and the non-dimensional Biot number is defined by $B i=H h_{0} / k$.

$\frac{\partial P^{(0)}}{\partial \zeta}=\frac{\partial^{2} W^{(0)}}{\partial \xi^{2}}+\frac{1}{\xi} \frac{\partial W^{(0)}}{\partial \xi}$,

$\frac{\partial^{2} \Theta^{(0)}}{\partial \xi^{2}}+\frac{1}{\xi} \frac{\partial \Theta^{(0)}}{\partial \xi}=0$

and the boundary conditions are

$\xi=R: \quad U^{(0)}=0, \quad W^{(0)}=W_{0}$

$\Theta^{(0)}=\Theta^{\mathrm{s}}(\xi)$

$\xi=\eta^{(0)}(\zeta): \quad U^{(0)}=W^{(0)} \eta^{(0)^{\prime},}$,

$\frac{\partial W^{(0)}}{\partial \xi}=-\frac{\partial \Theta^{(0)}}{\partial \zeta}-\frac{\mathrm{d} \eta^{(0)}}{\mathrm{d} \zeta} \frac{\partial \Theta^{(0)}}{\partial \xi}$,

$P^{(0)}=\left(\frac{1}{C}+1-\Theta^{(0)}\right) \frac{1}{\eta^{(0)}}$,

The non-dimensional equations and boundary conditions show the relationships of the orders of magnitude, and the perturbation method may be applied by the expansion based on the small parameter $\varepsilon$. For the analysis of order of magnitude, it is required that

$$
\begin{gathered}
R e=O(1), \quad P e=O(1), \quad B i=O(1), \\
C=O\left(10^{-1}-10^{0}\right) .
\end{gathered}
$$

It is noted that, definitions of the Reynolds number and the Peclet number in (2.13) are $\varepsilon$ times smaller then the usual definitions, because the typical velocity $v_{*}$ is $\varepsilon$ times smaller than the usual typical thermocapillary velocity. The quantities may be expanded as follows.

$\zeta=0$

$$
\begin{aligned}
U^{(0)} & =U_{e}(\xi), \quad W^{(0)}=W_{e}(\xi), \quad \Theta^{(0)}=\Theta_{e}(\zeta), \\
\eta^{(0)} & =1 ;
\end{aligned}
$$$$
\zeta=1: \quad U^{(0)}=U_{l}, \quad W^{(0)}=W_{l}, \quad \Theta^{(0)}=\Theta_{l} .
$$

$\eta^{(0)}$ is written as $\eta$ hereafter for simplification. By using 
boundary condition, Eq. (3.11), the solution of Eq (3.6) is

$\Theta^{(0)}(\xi, \zeta)=\Theta^{\mathrm{s}}(\zeta)-\frac{B i\left(\Theta^{\mathrm{s}}-\Theta_{\mathrm{g}}\right) \eta}{1+B i \eta \ln (\eta / R)} \ln \left(\frac{\xi}{R}\right)$

The heat transfers from the solid at temperature $\Theta^{\mathrm{s}}$ to the free surface at temperature $\Theta^{(0)}(\eta, \zeta)$ and then to the gas at temperature $\Theta_{\mathrm{g}}$. The temperature in the liquid film will be uniform if the temperatures at solid boundary $\xi=R$ and at exit of melt vessel $\zeta=0$ keep uniform, same as the environmental gas temperature. In this case, there will be no thermocapillary flow in the liquid layer.

Eq. (3.4) implies that the pressure is a function of $\zeta$ only, and then Eq. (3.10) at free surface gives

$P^{(0)}(\zeta)=\frac{1}{\eta}\left[\frac{C+1}{C}-\Theta^{\mathrm{s}}(\zeta)+\frac{B i\left(\Theta^{\mathrm{s}}-\Theta_{\mathrm{g}}\right) \eta}{1+B i \eta \ln (\eta / R)}\right]$

Substituting Eq. (3.15) into Eqs. (3.3) and (3.5) and using free surface condition, Eq. (3.8), the velocity field in the liquid layer can be written as

$U^{(0)}=\frac{\mathrm{d}^{2} P^{(0)}}{\mathrm{d} \zeta^{2}} \frac{\left(\xi^{2}-R^{2}\right)^{2}}{16 \xi}-\frac{\mathrm{d} A}{\mathrm{~d} \zeta}\left[\frac{\xi^{2}}{2} \ln \left(\frac{\xi}{R}\right)-\frac{\xi^{2}-R^{2}}{4}\right]$,

$W^{(0)}=\frac{\mathrm{d} P^{(0)}}{\mathrm{d} \zeta} \frac{\xi^{2}-R^{2}}{2}+A(\zeta) \ln \left(\frac{\xi}{R}\right)+W_{0}$,

and the function $A(\zeta)$ is

$A(\zeta)=-\frac{\mathrm{d} P^{(0)}}{\mathrm{d} \zeta} \eta^{2}-\left[D(\zeta) \eta^{\prime}+\frac{\mathrm{d} \Theta^{\mathrm{s}}}{\mathrm{d} \zeta} \eta+\frac{\mathrm{d} D}{\mathrm{~d} \xi} \eta \ln \left(\frac{\eta}{R}\right)\right]$

where

$D(\zeta)=-\frac{B i\left(\Theta^{\mathrm{s}}-\Theta_{\mathrm{g}}\right) \eta}{1+B i \eta \ln (\eta h / R)}$

Eq. (3.17) shows that longitude velocity consists of a main jet velocity, $W_{0}$, in addition to the contribution of the cross-section variation and the thermocapillary effect. By using solutions (3.16) and (3.17), a stream function $\Psi(\xi, \zeta)$ can be easily obtained as

$$
\begin{aligned}
\Psi^{(0)}(\xi, \zeta)= & -\frac{\mathrm{d} P^{(0)}}{\mathrm{d} \zeta} \frac{\left(\xi^{2}-R^{2}\right)^{2}}{16} \\
& -A(\zeta)\left[\frac{\xi^{2}}{2} \ln \left(\frac{\xi}{R}\right)-\frac{\xi^{2}-R^{2}}{4}\right] \\
& -\frac{W_{0}}{2} \xi^{2}+\Psi_{0},
\end{aligned}
$$

where $\Psi_{0}$ is an integral constant and may be selected as zero. Eqs. (3.14)-(3.17) depend on the height of liquid layer, which is determined by boundary condition (3.9).

The velocity and temperature boundary conditions in (3.12) and (3.13) cannot be given arbitrarily, and must satisfy the distributions of general solutions (3.14), (3.16) and (3.17).

\section{Radii equation and its solution}

Substituting Eqs. (3.14), (3.16) and (3.17) into boundary condition, Eq. (3.9), at the free surface, the height equation of the liquid layer is given as

$$
\begin{aligned}
& \left\{\frac{\mathrm{d} P^{(0)}}{\mathrm{d} \zeta}\left[\frac{\eta^{2}-R^{2}}{4}-\frac{\eta^{2}}{2} \ln \left(\frac{\eta}{R}\right)\right]\right. \\
& \left.\quad-\left[D(\zeta) \eta^{\prime}+\frac{\mathrm{d} \Theta^{\mathrm{s}}}{\mathrm{d} \zeta} \eta+\frac{\mathrm{d} D}{\mathrm{~d} \zeta} \eta \ln \left(\frac{\eta}{R}\right)\right] \ln \left(\frac{\eta}{R}\right)+W_{0}\right\} \eta^{\prime} \\
& =\frac{\mathrm{d}^{2} P^{(0)}}{\mathrm{d} \zeta^{2}} \frac{\left(\eta^{2}-R^{2}\right)^{2}}{16 \xi}-\frac{\mathrm{d} A}{\mathrm{~d} \zeta}\left[\frac{\eta^{2}}{2} \ln \left(\frac{\eta}{R}\right)-\frac{\eta^{2}-R^{2}}{4}\right] .
\end{aligned}
$$

According to the solution of pressure (3.15) and definition (3.18), Eq. (4.1) of the height $\eta$ is a secondorder ordinary differential equation of variable $\zeta$ and may be written as

$F\left(\eta, \eta^{\prime}, \zeta\right) \eta^{\prime \prime}=G\left(\eta, \eta^{\prime}, \zeta\right)$,

where

$$
\begin{aligned}
F(\eta, \zeta)= & {\left[\frac{\eta^{2}}{2} \ln \left(\frac{\eta}{R}\right)-\frac{\eta^{2}-R^{2}}{4}\right] D(\zeta) } \\
& -\left[\eta^{3} \ln \left(\frac{\eta}{R}\right)-\frac{\left(\eta^{2}-R^{2}\right)\left(8 \eta^{3}+\eta^{2}-R^{2}\right)}{16 \eta^{2}}\right] \\
& \times \frac{B i\left(\Theta^{\mathrm{s}}-\Theta_{\mathrm{g}}\right)(1-B i \eta)}{[1+B i \eta \ln (\eta / R)]^{2}} \\
& -\left[\frac{\eta^{2}}{2} \ln \left(\frac{\eta}{R}\right)-\frac{\left(\eta^{2}-R^{2}\right)\left(4 \eta^{3}+\eta^{2}-R^{2}\right)}{16 \eta^{3}}\right] \\
& \times\left\{\frac{1}{C}+1-\Theta^{\mathrm{s}}-\left[1-\ln \left(\frac{\eta}{R}\right)\right] D(\zeta)\right\},
\end{aligned}
$$


and

$$
\begin{aligned}
G\left(\eta, \eta^{\prime}, \zeta\right)= & G 1\left(\eta, \eta^{\prime}, \zeta\right) G 2\left(\eta, \eta^{\prime}\right) \\
& +G 3\left(\eta, \eta^{\prime}, \zeta\right) G 4(\eta)+G 5\left(\eta, \eta^{\prime}, \zeta\right) \\
& +G 6\left(\eta, \eta^{\prime}, \zeta\right) G 7(\eta) \\
& +G 8\left(\eta, \eta^{\prime}, \zeta\right) G 9(\eta),
\end{aligned}
$$

where

$$
\begin{aligned}
& G 1=\left\{\left[\ln \left(\frac{\eta}{R}\right)\right]^{2} \frac{\mathrm{d} D}{\mathrm{~d} \zeta}-\ln \left(\frac{\eta}{R}\right) \eta^{\prime} D+\eta \ln \left(\frac{\eta}{R}\right) \frac{\mathrm{d} \Theta^{\mathrm{s}}}{\mathrm{d} \zeta}\right. \\
& \left.+W_{0}\right\} \eta^{\prime} \\
& G 2=\left[\frac{\eta}{2} \ln \left(\frac{\eta}{R}\right)-\frac{\eta^{2}-R^{2}}{4}\right]\left\{\left[2+\ln \left(\frac{\eta}{R}\right)\right] \eta^{\prime} \frac{\mathrm{d} D}{\mathrm{~d} \zeta}\right. \\
& \left.+\frac{\mathrm{d}^{2} \Theta^{\mathrm{s}}}{\mathrm{d} \zeta^{2}} \eta+\frac{\mathrm{d} \Theta^{\mathrm{s}}}{\mathrm{d} \zeta} \eta^{\prime}\right\} \\
& G 3=\left[\frac{\left(\eta^{2}-R^{2}\right)(1+2 \eta)}{4}-\left(\eta^{3}+\frac{\eta}{2}\right) \ln \left(\frac{\eta}{R}\right)\right] \\
& \times\left\{\frac{1}{\eta} \ln \left(\frac{\eta}{R}\right) \frac{\mathrm{d} D}{\mathrm{~d} \zeta}-\frac{1}{\eta} \frac{\mathrm{d} \Theta^{\mathrm{s}}}{\mathrm{d} \zeta}\right. \\
& \left.-\frac{\frac{1}{c}+1-\Theta^{\mathrm{s}}-\left[1-\ln \left(\frac{\eta}{R}\right)\right] D}{\eta^{2}} \eta^{\prime}\right\} \eta^{\prime}, \\
& G 4=\left\{\frac{2\left[1-\ln \left(\frac{\eta}{R}\right)\right] \eta^{\prime}}{\eta^{2}} \frac{\mathrm{d} D}{\mathrm{~d} \zeta}\right. \\
& +2 \frac{\frac{1}{C}+1-\Theta^{\mathrm{s}}-\left[1.5-\ln \left(\frac{\eta}{R}\right)\right] D}{\eta^{3}} \eta^{\prime 2}-\frac{1}{\eta} \frac{\mathrm{d}^{2} \Theta^{\mathrm{s}}}{\mathrm{d} \zeta^{2}} \\
& \left.+\frac{2 \eta^{\prime}}{\eta^{2}} \frac{\mathrm{d} \Theta^{\mathrm{s}}}{\mathrm{d} \zeta}\right\} \cdot\left[\frac{\eta^{4}}{2} \ln \left(\frac{\eta}{R}\right)-\frac{5 \eta^{2}-R^{2}}{16}\right],
\end{aligned}
$$

$$
\begin{aligned}
G 5= & {\left[\eta^{3} \ln \left(\frac{\eta}{R}\right)\right.} \\
& \left.-\frac{\left(\eta^{2}-R^{2}\right)\left(8 \eta^{3}+\eta^{2}-R^{2}\right)}{16 \eta^{2}}\right] G 6\left(\eta, \eta^{\prime}, \zeta\right), \\
G 6\left(\eta, \eta^{\prime}, \zeta\right)=\frac{B i\left[\frac{\mathrm{d} \Theta^{\mathrm{s}}}{\mathrm{d} \zeta} \eta^{\prime}+\frac{\mathrm{d}^{2} \Theta^{\mathrm{s}}}{\mathrm{d} \zeta^{2}} \eta\right]}{1+B i \eta \ln (\eta / R)} & \\
+ & \frac{2 B i^{2}\left(\Theta^{\mathrm{s}}-\Theta_{\mathrm{g}}\right)(1-B i \eta)[1+\ln (\eta / R)]}{[1+B i \eta \ln (\eta / R)]^{3}} \\
- & \frac{B i^{2}\left(\Theta^{\mathrm{s}}-\Theta_{\mathrm{g}}\right) \eta^{\prime}+B i[-1+B i \eta+\eta+\eta \ln (\eta / R)]}{[1+B i \eta \ln (\eta / R)]^{2}}
\end{aligned}
$$

Eq. (4.2) requires two boundary conditions of $\eta$, which are usually given as

$\eta(0)=1, \quad \frac{\mathrm{d} \eta(0)}{\mathrm{d} \zeta}=\gamma$,

where $\gamma$ is the expansive angle of the jet liquid on the free surface at the vessel exit.

According to temperature distribution (2.14), the non-dimensional temperature $\Theta^{\mathrm{s}}$ on the fiber boundary $\xi=R$ may be written as

$\Theta^{\mathrm{s}}=\mathbf{1}-\left(1-\Theta_{m}\right) \zeta$,

where non-dimensional temperature $\Theta_{m}=T_{m} / T_{*}$. The temperature on the solid fiber decreases uniformly from the vessel exit value $T_{*}(r)$ at $z=0$ to the temperature $T_{m}$ at $z=\ell$, because of the heat transfers from the relatively higher temperature in the liquid or melt to the relatively lower environmental temperature $T=T_{\mathrm{g}}$. Substituting temperature distribution (4.6), the solution of Eq. (4.2) under boundary conditions (4.5) may then be obtained. Typical parameters in the calculations are adopted as

$\Theta_{\mathrm{g}}=0.1, \quad \Theta_{m}=0.1, \quad B i=0.5$.

The longitudinal velocity $W_{0}$ will be from 0 to 9 , and capillary number $C$ is adopted in the range $[0.1,0.9]$.

Substituting Eq. (4.7) into temperature distribution equation (3.14), the temperature in the jet liquid layer may be demonstrated as

$\Theta^{(0)}(\xi, \zeta)=1-0.9 \zeta-\frac{0.45(1-\zeta) \eta}{1+0.5 \eta \ln (2 \eta)} \ln (2 \xi)$.

The first two terms on the right-hand side give the temperature distribution at the fiber boundary which is independent of the $\xi$, and the third term is the 
temperature decrease due to the heat transfer to the environmental gas. Relationship (4.8) gives a determined temperature distribution at the vessel exit $\Theta^{(0)}(0, \xi)=1-0.45 \ln (2 \xi) /(1+0.5 \ln 2)$ and an uniform temperature $\Theta^{(0)}(\xi, 1)=0.1$ at the cross-section $\zeta=1$. These are the reasonable boundary conditions required by Eqs. (3.12) and (3.13).

Similar discussions can be applied to the flow fields, which depend mainly on both thermocapillary effect and the jet flow. By using height equation (4.8), equation of the stream function (3.19) is reduced to

$$
\begin{aligned}
\Psi^{(0)}(\xi, \zeta)= & -0.9 \eta-\frac{0.45(1-\zeta) \eta \eta^{\prime}}{1+0.5 \eta \ln (2 \eta)} \\
& +\eta \ln (2 \eta)\left[\frac{0.45 \eta}{1+0.5 \eta \ln (2 \eta)}\right. \\
& \left.\left.-\frac{0.45(1-\zeta)(1-0.5 \eta)}{[1+0.5 \eta \ln (2 \eta)]^{2}} \eta^{\prime}\right]\right\} Q(\xi) \\
& +\left(-\frac{\mathrm{d} P}{\mathrm{~d} \zeta}\right)\left[\frac{\left(\xi^{2}-R^{2}\right)^{2}}{16}-\eta^{2} Q(\xi)\right] \\
& +\frac{W_{0}}{2} \xi^{2}+\Psi_{0},
\end{aligned}
$$

where the integral constant $\Psi_{0}$ in relationship (3.19) may be adopted as zero, and

$$
\begin{aligned}
-\frac{\mathrm{d} P^{(0)}}{\mathrm{d} \zeta}= & -\frac{0.9}{\eta}-\frac{0.45 \ln (2 \eta)}{1+0.5 \eta \ln (2 \eta)} \\
& +\frac{0.45(1-\zeta)(1-0.5 \eta) \ln (2 \eta)}{\eta[1+0.5 \eta \ln (2 \eta)]^{2}} \eta^{\prime} \\
& +\left\{\frac{1}{C}+0.9 \zeta-\frac{0.45(1-\zeta) \eta[1-\ln (2 \eta)]}{1+0.5 \eta \ln (2 \eta)}\right\} \frac{\eta^{\prime}}{\eta^{2}},
\end{aligned}
$$

$Q(\xi)=\frac{\xi^{2}}{2} \ln \left(\frac{\xi}{R}\right)-\frac{\xi^{2}-R^{2}}{4}$

The radii of liquid layer may be written as

$\eta^{(0)}(\zeta)=1+\delta(\zeta)$

and there are $|\delta(\zeta)| \ll 1$ in many cases. In this case, by using Eqs. (4.5) and (4.6), stream function (4.9) may be written as

$$
\begin{aligned}
\Psi^{(0)}(\xi, \zeta)= & -\left[0.070727\left(\xi^{2}-0.25\right)^{2}+0.5 W_{0} \xi^{2}-\Psi_{0}\right] \\
& +\left[16 / C+0.062659 \zeta-0.001033\left(\xi^{2}-0.25\right)^{2}\right. \\
& +(0.1290925+0.85691755 \zeta) Q(\xi)] \delta^{\prime} \\
& +\left[0.001033\left(\xi^{2}-0.25\right)^{2}+0.866932 Q(\xi)\right] \delta \\
& +O\left(\delta^{2}\right),
\end{aligned}
$$

where $\delta^{\prime}$ has the same order of magnitude as $\delta$. The first bracket on the right-hand side is the zero order stream function, which shows that the main flow is dominated by the jet velocity if the jet velocity is not smaller than the typical thermocapillary velocity $W_{0} \geq 1$. In this case, the thermocapillary flow is secondary. The term of $C$ shows the influence of surface tension, which is involved in second bracket of the first order relationship.

\section{The cross-section profiles}

In case of pure thermocapillary convection, there is no jet flow $W_{0}=0$, and the flow is driven by the gradient of surface tension due to the temperature non-uniformity on the free surface. This case is similar to the one discussed in [5,6]. Fig. 2 shows profiles of liquid radii depending on the capillary number $C$ for case $W_{0}=0$ and $\gamma=0.5$. The results show that the larger the capillary number, the larger the radii of liquid layer. Relationship (3.15) gives the results that, the larger Capillary number $C$ relates to a smaller pressure, and a larger radius of liquid column due to the momentum equilibrium on the free surface in the normal direction.

However, the conclusion is reversed if the jet velocity is larger than the typical thermocapillary velocity

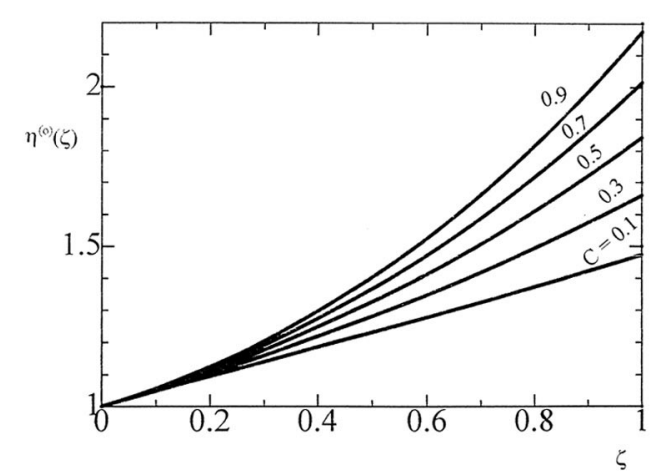

Fig. 2. The radii profiles of liquid column depending on Capillary number $C=0.9,0.7,0.5,0.3,0.1$ (from upper to lower) in pure thermocapillary convection $W_{0}=0$ and $\gamma=0.5$. 


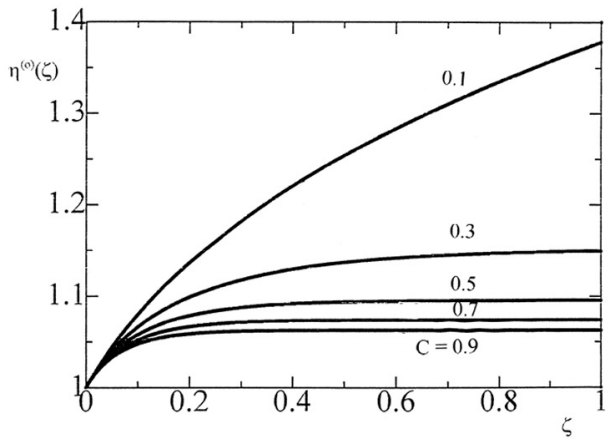

Fig. 3. The radii profiles of liquid column depending on Capillary number $C=0.1,0.3,0.5,0.7,0.9$ (from upper to lower) in case of $W_{0}=5$ and $\gamma=1.0$.

as shown in Fig. 3, where $W_{0}=5$. In this case, the pressure distribution is determined mainly by the effect of jet flow but not by the thermocapillary flow. The larger capillary number $C$ relates to a smaller surface tension $\sigma$, and then a smaller radius of the liquid column. The calculations show that the radii of liquid column is increased with increase of the Capillary number $C$ if the jet velocity is not larger than the typical thermocapillary velocity, that is, $W_{0} \leq 1$, otherwise, the radii will be decreased with the increasing of the Capillary number $C$.

The expanding angle $\gamma$ at the vessel exit $\zeta=0$ is a sensitive parameter as shown in Fig. 4 for case $W_{0}=5$ and $C=0.5$. As usually expected, the larger the expanding angle, the larger the radius of liquid column. It should be noted that the non-dimensional relationship of the second, boundary condition in (4.5)

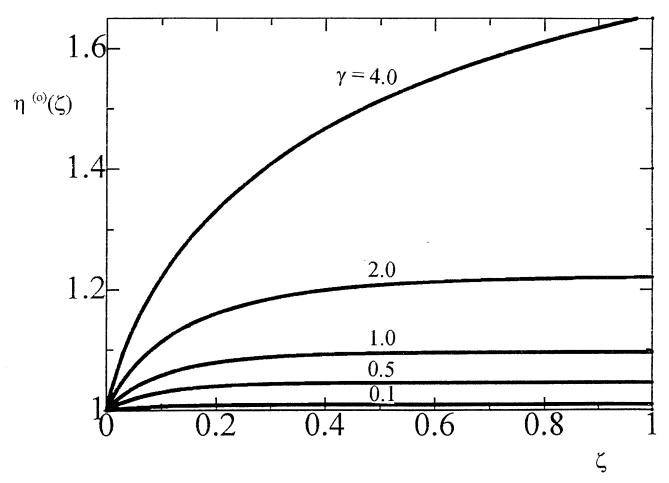

Fig. 4. Influence of the expending angle $\gamma$ on the radii profiles of the jet liquid column for cases $W_{0}=5$ and $C=0.5$. The profiles relate to $\gamma=4.0,2.0,1.0,0.5$ and 0.1 , respectively, from upper to lower.

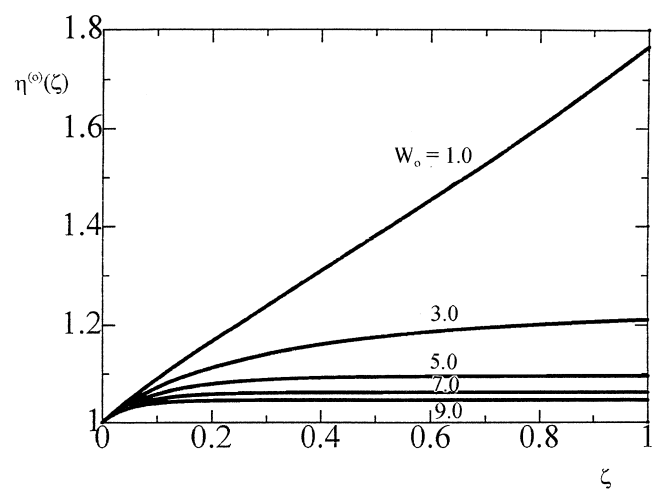

Fig. 5. Influence of jet velocities on the radii profiles of the jet liquid column for case $\gamma=1.0$ and $C=0.5$. The profiles relate to $W_{0}=1.0,3.0,5.0,7.0$ and 9.0 , respectively, from upper to lower.

can be written in the dimensional form as

$\frac{\mathrm{d} h(0)}{\mathrm{d} z}=\varepsilon \gamma$.

Therefore, the real expanding angle $\tan ^{-1}(\varepsilon \gamma)$ is a smaller angle and close to zero in the cases discussed here. The influence of jet velocity $W_{0}$ is given in Fig. 5 . The larger the jet velocity, the smaller the radius of liquid column. This conclusion agrees with the one discussed for Fig. 3.

A general conclusion on the effect of thermocapillary flow on the enlargement of cross-section of jet liquid is summarized in Table 1, where the liquid column height $\eta^{(0)}(\xi, 1)=$ constant, depending on $W_{0}$ and $\gamma$ are given. The results show that the cross-section may be increased several times in case of larger expanding angle $\gamma$ and smaller jet velocity $W_{0}$.

\section{Discussions}

The thermocapillary flow in an annular liquid film

Table 1

Liquid column radius $\eta^{(0)}(\xi, 1)$ depending on $W_{0}$ and $\gamma$ in case of $C=0.5$

\begin{tabular}{lllllll}
\hline$\gamma$ & \multicolumn{1}{l}{$W_{0}$} & & & & & \\
\cline { 2 - 7 } & 0 & 0.1 & 0.5 & 1.0 & 2.0 & 5.0 \\
\hline 0.1 & 1.2774 & 1.2480 & 1.1555 & 1.0860 & 1.0321 & 1.0087 \\
0.5 & 1.8437 & 1.7855 & 1.5825 & 1.3912 & 1.1760 & 1.0451 \\
1.0 & 2.4197 & 2.3402 & 2.0545 & 1.7669 & 1.3908 & 1.0954 \\
\hline
\end{tabular}


painted on a moving fiber was discussed by using the lubrication approximation and the perturbation method. The analytical solutions of pressure, temperature and velocities were obtained in the zero order. The variation of liquid column radii is determined by a second order ordinary differential equation, and may be solved by two conditions of radius at the vessel exit $z=0$. By using the Runge-Kutta method, the problem was solved. The variation of liquid radii depending on the thermocapillary flow, in addition of the jet velocity $W_{0}$, the Capillary number $C$ and the expanding angle were analyzed. The special case $W_{0}=0$ relates to the case discussed in $[5,6]$. However, the configuration is cylindrical in the present paper.

The approximations of lubrication theory and perturbation theory have of course limitations. The Barus effect may has larger variations of cross-section, which may be two or three times in some cases. Based on the results of present paper, a large variation of the crosssection may be obtained in case of relatively large expanding angle and relatively smaller jet velocity as shown in Table 1.

The results of present paper show mainly the mechanism of the cross-section variation due to the thermocapillary effect and jet flow. Firstly, the solidification is important from the point of view of processing. The temperature will be decreased along the liquid fiber and the melt will be solidified at a distance where the liquid temperature equals the melt point due to the heat transfer. The solidification is not involved in the present paper, and the cross-section $z=l$ is selected to be located before the solidification. Secondly, The fiber processing requires more complex configuration and wider parameter regime, and an ideal model is used in the present paper. However, the main purpose of the present paper concentrates in the mechanism study to show the existence of the thermocapillary flow induced by the heat transfer. Many effects should be included based on the processing process, and should be studied in the future.

\section{Acknowledgements}

W.R. Hu, acknowledges the kind invitation of Prof. Y. Sugioka, the President of the Kyushu University and the kind hospitality and collaboration with Prof. N. Imaishi for him to be a visiting Professor in the Institute of Advanced Material Study of the Kyushu University. This research is done mostly during his visiting period in the Kyushu University, and was completed when he returned to Beijing. The research is supported in part by the Project 95-Yu-34 of the Ministry of Science and Technology of China and grant 19789201 of the National Natural Science Foundation of China.

\section{References}

[1] D. Quere, Fluid coating on a fiber, Ann. Rev. Fluid Mechanics 31 (1999) 347.

[2] C.L. Tucker, Computer Modeling for Polymer Processing, Hanser, New York, 1989.

[3] W.A. Gifford, The effect of surface tension on extrudate swell from square and rectangular channels, Polymer Engineering and Science 38 (1998) 1167.

[4] O.O. Ramdaul, D. Quere, Thickening factor in Marangoni coating, Langmuir 13 (1997) 2911.

[5] S.M. Pimputkar, S. Ostrach, Transient thermocapillary flow in the thin liquid layers, Physics Fluids 23 (1980) 1281.

[6] D.L. Hitt, M.K. Smith, Radiation-driven thermocapillary flows in optically thick liquid films, Physics Fluids A5 (1993) 2624.

[7] W.R. Hu, N. Imaishi, Thermocapillary flow in a jet of liquid film painted in a moving boundary, Langmuir 16 (2000) 4632. 